Supplement of Sci. Dril., 29, 19-37, 2021

https://doi.org/10.5194/sd-29-19-2021-supplement

(c) Author(s) 2021. CC BY 4.0 License.

(c) (1)

Supplement of

\title{
Composite development and stratigraphy of the Onepoto maar lake sediment sequence (Auckland Volcanic Field, New Zealand)
}

Benjamin Läuchli et al.

Correspondence to: Benjamin Läuchli (bluc030@aucklanduni.ac.nz)

The copyright of individual parts of the supplement might differ from the article licence. 
Table S1: Core section lengths with rates of expansion and adjusted depths for composite record build.

\begin{tabular}{|c|c|c|c|c|c|c|c|}
\hline $\begin{array}{l}\text { Bore- } \\
\text { hole }\end{array}$ & Section No. & $\begin{array}{l}\text { Length after } \\
\text { sediments } \\
\text { extruded } \\
\text { into PVC } \\
\text { tubes }[\mathrm{cm}]\end{array}$ & $\begin{array}{l}\text { Drill } \\
\text { Rod } \\
\text { Length } \\
{[\mathrm{cm}]}\end{array}$ & $\begin{array}{l}\text { Expansion } \\
\text { by }(\%)\end{array}$ & $\begin{array}{l}\text { Adjusted } \\
\text { Composite } \\
\text { Base Depth } \\
{[\mathrm{m}]}\end{array}$ & $\begin{array}{l}\text { Adjusted } \\
\text { Composite } \\
\text { Top Depth } \\
\text { [m] }\end{array}$ & Comment \\
\hline A & $\begin{array}{l}\text { One_18A_39.6- } \\
41.1 \mathrm{~m}\end{array}$ & 173 & 150 & 15.33 & 41.14 & 39.64 & \\
\hline A & $\begin{array}{l}\text { One_18A_41.1- } \\
42.7 \mathrm{~m}\end{array}$ & 170 & 160 & 6.25 & 42,77 & 41.14 & \\
\hline A & $\begin{array}{l}\text { One_18A_42.7- } \\
44.2 \mathrm{~m}\end{array}$ & -- & -- & -- & -- & -- & $\begin{array}{l}\text { Disturbed/core } \\
\text { loss }\end{array}$ \\
\hline A & $\begin{array}{l}\text { One_18A_44.2- } \\
46.7 \mathrm{~m}\end{array}$ & 158 & 150 & -- & -- & -- & $\begin{array}{l}\text { Disturbed/core } \\
\text { loss }\end{array}$ \\
\hline A & $\begin{array}{l}\text { One_18A_46.7- } \\
48.3 \mathrm{~m}\end{array}$ & 203 & 160 & 26.88 & 48.32 & 46.72 & \\
\hline A & $\begin{array}{l}\text { One_18A_48.3- } \\
49.8 \mathrm{~m}\end{array}$ & 193 & 150 & 28.66 & 49.82 & 48.32 & \\
\hline A & $\begin{array}{l}\text { One_18A_49.8- } \\
51.4 \mathrm{~m}\end{array}$ & 214 & 160 & 33.75 & 51.42 & 49.82 & \\
\hline A & $\begin{array}{l}\text { One_18A_51.4- } \\
52.9 \mathrm{~m}\end{array}$ & 205 & 150 & 36.67 & 52.93 & 51.42 & \\
\hline A & $\begin{array}{l}\text { One_18A_52.9- } \\
54.4 \mathrm{~m}\end{array}$ & 208 & 150 & 30 & 54.43 & 52.93 & \\
\hline A & $\begin{array}{l}\text { One_18A_54.4- } \\
56.0 \mathrm{~m}\end{array}$ & 169 & 160 & 5.63 & 56.15 & 55.04 & \\
\hline A & $\begin{array}{l}\text { One_18A_56.0- } \\
57.5 \mathrm{~m}\end{array}$ & 263 & 150 & 75.33 & 59.62 & 57.79 & \\
\hline A & $\begin{array}{l}\text { One_18A_57.5- } \\
59.0 \mathrm{~m}\end{array}$ & 187 & 150 & 24.67 & 61.12 & 59.62 & \\
\hline A & $\begin{array}{l}\text { One_18A_59.0- } \\
60.5 \mathrm{~m}\end{array}$ & 224 & 150 & 49.33 & 62.62 & 61.12 & \\
\hline A & $\begin{array}{l}\text { One_18A_60.5- } \\
62.1 \mathrm{~m}\end{array}$ & 194.5 & 160 & 27.81 & 64.40 & 62.64 & \\
\hline A & $\begin{array}{l}\text { One_18A_62.1- } \\
63.3 \mathrm{~m}\end{array}$ & 185 & 150 & 23.33 & 65.67 & 64.60 & \\
\hline A & $\begin{array}{l}\text { One_18A_63.6- } \\
65.1 \mathrm{~m}\end{array}$ & 175 & 150 & 16.67 & 67.31 & 65.80 & \\
\hline A & $\begin{array}{l}\text { One_18A_66.6- } \\
68.2 \mathrm{~m}\end{array}$ & 146 & 160 & -- & 68.86 & 67.40 & $\begin{array}{l}\text { Corrected for } \\
\text { slumped } \\
\text { material }\end{array}$ \\
\hline A & $\begin{array}{l}\text { One_18A_68.2- } \\
69.7 \mathrm{~m}\end{array}$ & 168 & 150 & 12 & 70.37 & 68.87 & \\
\hline A & $\begin{array}{l}\text { One_18A_69.7- } \\
71.2 \mathrm{~m}\end{array}$ & 68 & 150 & -- & 72.27 & 71.59 & $\begin{array}{l}\text { Corrected for } \\
\text { slumped } \\
\text { material }\end{array}$ \\
\hline A & $\begin{array}{l}\text { One_18A_71.2- } \\
72.7 \mathrm{~m}\end{array}$ & 188 & 150 & 25.33 & 74.12 & 72.69 & \\
\hline B & $\begin{array}{l}\text { One_18B_39.6- } \\
41.6\end{array}$ & 191 & 200 & -- & 41.32 & 39.41 & $\begin{array}{l}\text { Top } 9 \mathrm{~cm} \\
\text { disturbed }\end{array}$ \\
\hline B & $\begin{array}{l}\text { One_18B_41.6- } \\
43.1 \mathrm{~m}\end{array}$ & 188 & 150 & 25.33 & 22.67 & & \\
\hline B & $\begin{array}{l}\text { One_18B_43.1- } \\
44.6 \mathrm{~m} \text { - }\end{array}$ & 213 & 150 & 42 & & & \\
\hline B & $\begin{array}{l}\text { One_18B_47.6- } \\
48.2\end{array}$ & -- & -- & -- & -- & -- & $\begin{array}{l}\text { Disturbed/core } \\
\text { loss }\end{array}$ \\
\hline B & $\begin{array}{l}\text { One_18B_48.3- } \\
50.3 \mathrm{~m}\end{array}$ & -- & -- & -- & -- & -- & $\begin{array}{l}\text { Disturbed/core } \\
\text { loss }\end{array}$ \\
\hline B & $\begin{array}{l}\text { One_18B_51.9- } \\
53.4 \mathrm{~m}\end{array}$ & 184 & 150 & 22.67 & 54.98 & 53.45 & \\
\hline B & $\begin{array}{l}\text { One_18B_53.4- } \\
54.9 \mathrm{~m}\end{array}$ & 176 & 150 & 17.33 & 56.54 & 55.05 & \\
\hline
\end{tabular}




\begin{tabular}{|c|c|c|c|c|c|c|c|}
\hline $\begin{array}{l}\text { Bore- } \\
\text { hole }\end{array}$ & Section No. & $\begin{array}{l}\text { Length after } \\
\text { sediments } \\
\text { extruded } \\
\text { into PVC } \\
\text { tubes }[\mathrm{cm}]\end{array}$ & $\begin{array}{l}\text { Drill } \\
\text { Rod } \\
\text { Length } \\
{[\mathrm{cm}]}\end{array}$ & $\begin{array}{l}\text { Expansion } \\
\text { by }(\%)\end{array}$ & $\begin{array}{l}\text { Adjusted } \\
\text { Composite } \\
\text { Base Depth } \\
{[\mathrm{m}]}\end{array}$ & $\begin{array}{l}\text { Adjusted } \\
\text { Composite } \\
\text { Top Depth } \\
{[\mathrm{m}]}\end{array}$ & Comment \\
\hline B & $\begin{array}{l}\text { One_18B_54.9- } \\
56.5 \mathrm{~m}\end{array}$ & 135 & 160 & -- & 57.90 & 56.55 & \\
\hline B & $\begin{array}{l}\text { One_18B_56.5- } \\
58.0 \mathrm{~m}\end{array}$ & 145 & 150 & -- & 59.55 & 58.10 & \\
\hline B & $\begin{array}{l}\text { One_18B_58.0- } \\
59.5 \mathrm{~m}\end{array}$ & 208.5 & 150 & 32.33 & 61.39 & 59.81 & \\
\hline B & $\begin{array}{l}\text { One_18B_59.5- } \\
61.0 \mathrm{~m}\end{array}$ & 209 & 150 & 39.33 & 63.08 & 61.58 & \\
\hline B & $\begin{array}{l}\text { One_18B_61.0- } \\
62.6 \mathrm{~m}\end{array}$ & 197 & 160 & 24.67 & 64.68 & 63.08 & \\
\hline B & $\begin{array}{l}\text { One_18B_64.1- } \\
65.6 \mathrm{~m}\end{array}$ & 157 & 150 & 4.67 & 67.67 & 66.18 & \\
\hline B & $\begin{array}{l}\text { One_18B_65.6- } \\
67.1 \mathrm{~m}\end{array}$ & 187 & 150 & 24.67 & 69.99 & 68.86 & \\
\hline B & $\begin{array}{l}\text { One_18B_67.1- } \\
68.7 \mathrm{~m}\end{array}$ & 162 & 160 & 1.33 & 70.48 & 69.99 & \\
\hline B & $\begin{array}{l}\text { One_18B_70.2- } \\
71.1 \mathrm{~m}\end{array}$ & 154 & 150 & -- & & & $\begin{array}{l}\text { Disturbed } \\
\text { sediments }\end{array}$ \\
\hline B & $\begin{array}{l}\text { One_18B_71.7- } \\
73.2 \mathrm{~m}\end{array}$ & 167 & 150 & -- & & & $\begin{array}{l}\text { Disturbed } \\
\text { sediments }\end{array}$ \\
\hline
\end{tabular}


Table S2: Glass Analysis or the 2018 Onepoto lake sediment core - reserved data repository doi $=10.6084 / \mathrm{m} 9$. figshare.13683658.

$\mathrm{Rm}=$ Rotoma; $\mathrm{Wh}=$ Waiohau; $\mathrm{Rr}=$ Rotorua; $\mathrm{Rw}=$ Rerewhakaaitu; Ok = Okareka; $\mathrm{Kw}=$ Kawkawa/Oruanui; $\mathrm{O}=\mathrm{Okaia} ; \mathrm{Mk}=\mathrm{Maketu}$; $\mathrm{Ta}=$ Tahuna; Re = Rotoehu; Eg = Egmont-sourced tephra

\begin{tabular}{|c|c|c|c|c|c|c|c|c|c|c|c|c|c|}
\hline $\begin{array}{l}\text { Tephra } \\
\text { Name }\end{array}$ & $\begin{array}{l}\text { Bore- } \\
\text { hole } \\
\text { A }\end{array}$ & $\mathrm{SiO}_{2}$ & $\mathrm{TiO}_{2}$ & $\mathrm{Al}_{2} \mathrm{O}_{3}$ & $\mathrm{FeO}$ & MnO & MgO & $\mathrm{CaO}$ & $\mathrm{Na}_{2} \mathrm{O}$ & $\mathrm{K}_{2} \mathrm{O}$ & Cl & Total & $\begin{array}{l}\text { Sample } \\
\text { ID }\end{array}$ \\
\hline $\mathrm{Rm}$ & $\begin{array}{l}\text { Core } \\
\text { A }\end{array}$ & 78.39 & 0.10 & 12.43 & 0.95 & 0.10 & 0.06 & 0.64 & 3.87 & 3.46 & 0.17 & 0.82 & At_2.1 \\
\hline $\mathrm{Rm}$ & $\begin{array}{l}\text { core } \\
\text { A }\end{array}$ & 78.03 & 0.12 & 12.58 & 0.94 & 0.08 & 0.15 & 0.67 & 3.87 & 3.57 & 0.13 & 1.23 & At_2.2 \\
\hline $\mathrm{Rm}$ & $\begin{array}{l}\text { core } \\
\text { A }\end{array}$ & 77.94 & 0.11 & 12.56 & 1.07 & 0.07 & 0.14 & 0.71 & 4.10 & 3.30 & 0.17 & 0.16 & At_2.3 \\
\hline $\mathrm{Rm}$ & $\begin{array}{l}\text { core } \\
\text { A }\end{array}$ & 78.01 & 0.13 & 12.64 & 0.80 & 0.07 & 0.13 & 0.68 & 4.10 & 3.43 & 0.13 & 0.79 & At_2.4 \\
\hline $\mathrm{Rm}$ & $\begin{array}{l}\text { core } \\
\text { A }\end{array}$ & 78.18 & 0.14 & 12.55 & 0.84 & 0.05 & 0.13 & 0.70 & 3.86 & 3.55 & 0.15 & 2.71 & At_2.6 \\
\hline $\mathrm{Rm}$ & $\begin{array}{l}\text { core } \\
\text { A }\end{array}$ & 77.96 & 0.16 & 12.50 & 0.77 & 0.04 & 0.14 & 0.67 & 4.14 & 3.62 & 0.19 & 3.65 & At_2.7 \\
\hline $\mathrm{Rm}$ & $\begin{array}{l}\text { core } \\
\text { A }\end{array}$ & 78.38 & 0.12 & 12.46 & 0.84 & 0.08 & 0.11 & 0.70 & 3.87 & 3.44 & 0.14 & 3.34 & At_2.8 \\
\hline $\mathrm{Rm}$ & $\begin{array}{l}\text { core } \\
\text { A }\end{array}$ & 78.10 & 0.13 & 12.49 & 0.83 & 0.08 & 0.14 & 0.68 & 3.99 & 3.55 & 0.14 & 2.19 & At_2.9 \\
\hline $\mathrm{Rm}$ & $\begin{array}{l}\text { core } \\
\text { A }\end{array}$ & 78.04 & 0.12 & 12.67 & 0.94 & 0.04 & 0.11 & 0.67 & 3.93 & 3.48 & 0.15 & 0.94 & At_2.10 \\
\hline $\mathrm{Rm}$ & $\begin{array}{l}\text { core } \\
\text { A }\end{array}$ & 78.26 & 0.10 & 12.55 & 0.87 & 0.05 & 0.12 & 0.73 & 3.90 & 3.43 & 0.17 & 0.45 & At_2.11 \\
\hline $\mathrm{Rm}$ & $\begin{array}{l}\text { core } \\
\text { A }\end{array}$ & 78.22 & 0.12 & 12.58 & 0.93 & 0.04 & 0.13 & 0.69 & 4.01 & 3.29 & 0.18 & 2.95 & At_2.13 \\
\hline $\mathrm{Rm}$ & $\begin{array}{l}\text { core } \\
\text { A }\end{array}$ & 78.14 & 0.14 & 12.49 & 0.92 & 0.08 & 0.14 & 0.66 & 4.03 & 3.39 & 0.16 & 3.23 & At_2.15 \\
\hline $\mathrm{Rm}$ & $\begin{array}{l}\text { core } \\
\text { A }\end{array}$ & 78.07 & 0.13 & 12.76 & 0.92 & 0.05 & 0.12 & 0.67 & 3.94 & 3.34 & 0.16 & 3.13 & At_2.16 \\
\hline $\mathrm{Rm}$ & $\begin{array}{l}\text { core } \\
\text { A }\end{array}$ & 78.19 & 0.15 & 12.59 & 0.81 & 0.06 & 0.13 & 0.70 & 4.01 & 3.36 & 0.15 & 0.67 & At_2.17 \\
\hline $\mathrm{Rm}$ & $\begin{array}{l}\text { core } \\
\text { A }\end{array}$ & 78.08 & 0.11 & 12.58 & 0.82 & 0.07 & 0.14 & 0.70 & 4.16 & 3.34 & 0.16 & 1.08 & At_2.18 \\
\hline $\mathrm{Rm}$ & $\begin{array}{l}\text { core } \\
\text { A }\end{array}$ & 78.16 & 0.08 & 12.66 & 0.82 & 0.05 & 0.11 & 0.70 & 3.98 & 3.42 & 0.18 & 0.66 & At_2.19 \\
\hline $\mathrm{Rm}$ & $\begin{array}{l}\text { core } \\
\text { A }\end{array}$ & 78.10 & 0.08 & 12.61 & 0.86 & 0.04 & 0.13 & 0.68 & 4.05 & 3.46 & 0.15 & 0.45 & At_2.20 \\
\hline $\mathrm{Rm}$ & $\begin{array}{l}\text { core } \\
\text { A }\end{array}$ & 77.96 & 0.11 & 12.65 & 0.86 & 0.07 & 0.17 & 0.69 & 4.11 & 3.38 & 0.19 & 3.36 & At_1.5 \\
\hline $\mathrm{Rm}$ & $\begin{array}{l}\text { core } \\
\text { A }\end{array}$ & 78.31 & 0.12 & 12.52 & 0.92 & 0.03 & 0.13 & 0.69 & 3.77 & 3.51 & 0.14 & 0.09 & At_1.8 \\
\hline $\mathrm{Rm}$ & $\begin{array}{l}\text { core } \\
\text { A }\end{array}$ & 78.20 & 0.10 & 12.66 & 0.86 & 0.09 & 0.12 & 0.69 & 3.89 & 3.38 & 0.16 & 1.54 & At_1.9 \\
\hline $\mathrm{Rm}$ & $\begin{array}{l}\text { core } \\
\text { A }\end{array}$ & 78.32 & 0.11 & 12.52 & 0.90 & 0.04 & 0.14 & 0.67 & 3.87 & 3.43 & 0.15 & 0.07 & At_1.11 \\
\hline $\mathrm{Rm}$ & $\begin{array}{l}\text { core } \\
\text { A }\end{array}$ & 78.14 & 0.13 & 12.63 & 1.01 & 0.05 & 0.13 & 0.66 & 4.02 & 3.23 & 0.17 & 0.99 & At_1.13 \\
\hline $\mathrm{Rm}$ & $\begin{array}{l}\text { core } \\
\text { A }\end{array}$ & 78.14 & 0.11 & 12.64 & 0.90 & 0.06 & 0.12 & 0.70 & 3.98 & 3.35 & 0.14 & 2.21 & At_1.14 \\
\hline $\mathrm{Rm}$ & $\begin{array}{l}\text { core } \\
\text { A }\end{array}$ & 77.74 & 0.14 & 12.56 & 0.79 & 0.07 & 0.16 & 0.64 & 4.17 & 3.73 & 0.15 & 1.27 & At_1.15 \\
\hline $\mathrm{Rm}$ & $\begin{array}{l}\text { core } \\
\text { B }\end{array}$ & 78.11 & 0.16 & 12.52 & 0.87 & 0.04 & 0.16 & 0.71 & 3.99 & 3.45 & 0.15 & 1.89 & At_1.17 \\
\hline $\mathrm{Rm}$ & $\begin{array}{l}\text { Core } \\
\text { B }\end{array}$ & 79.10 & 0.12 & 12.65 & 0.80 & 0.05 & 0.14 & 0.72 & 2.98 & 3.45 & 0.16 & 5.09 & Bt_2.1 \\
\hline $\mathrm{Rm}$ & $\begin{array}{l}\text { Core } \\
\text { B }\end{array}$ & 78.04 & 0.12 & 12.49 & 0.94 & 0.05 & 0.11 & 0.74 & 4.22 & 3.28 & 0.15 & 4.39 & Bt_2.4 \\
\hline $\mathrm{Rm}$ & Core & 78.03 & 0.11 & 12.54 & 0.64 & 0.09 & 0.10 & 0.69 & 4.36 & 3.45 & 0.23 & 3.84 & Bt_2.5 \\
\hline
\end{tabular}


Tephra Bore-

$\mathrm{SiO}_{2}$

Core $\quad 77.58$ B

$\mathrm{Rm}$

$\mathrm{Rm}$

$\mathrm{Rm}$

$\mathrm{Rm}$

$\mathrm{Rm}$

$\mathrm{Rm}$

$\mathrm{Rm}$

$\mathrm{Rm}$

$\mathrm{Rm}$

$\mathrm{Rm}$

$\mathrm{Rm}$

$\mathrm{Rm}$

Rm

$\mathrm{Rm}$

$\mathrm{Rm}$

$\mathrm{Rm}$

$\mathrm{Rm}$

$\mathrm{Rm}$

$\mathrm{Rm}$

$\mathrm{Rm}$

Rm

$\mathrm{Rm}$

Rm

$\mathrm{Rm}$

Rm

$\mathrm{Rm}$

Wh

Wh

Wh

Wh B

Core 77.91

B

Core $\quad 77.89$ B

Core $\quad 77.8$

B

77.85

Core $\quad 78.07$

$$
\text { B }
$$

Core $\quad 77.90$

B

Core $\quad 77.94$

$$
\text { B }
$$

Core $\quad 77.9$

B

B

B

77.95

$$
\text { B }
$$

B

Core 78.11

Core

B

Core $\quad 77.8$

Core $\quad 77.7$

B

Core 77.96 B

Core $\quad 77.82$

B

Core 77.8

$$
\text { B }
$$

B

Core $\quad 77.58$ B

Core $\quad 77.93$ Core

\section{B}

Core $78.04 \quad 0$.

Core $\quad 77.99 \quad 0.1$

B

Core $77.93 \quad 0.16$

B

Core $\quad 78.19 \quad 0.12$ $\begin{array}{lllll}\text { Core } & 78.04 & 0.11 & 12.53 & 0.83\end{array}$ B

Core $\quad 77.75$ B

Core $\quad 77.89$ B

Core $\quad 77.69$ B

Core $\quad 77.58$ B

Core $\quad 78.00$

$\begin{array}{lll}0.13 & 12.59 & 0.99\end{array}$

$0.11 \quad 12.66 \quad 0.86$

$\begin{array}{lll}0.12 & 12.43 \quad 0.80\end{array}$

$0.12 \quad 12.51 \quad 0.90$

$\begin{array}{lll}0.10 & 12.53 \quad 0.75\end{array}$

$\begin{array}{lll}0.13 & 12.51 \quad 0.93\end{array}$

0.15

\begin{abstract}
12.59
\end{abstract}
2.590 .82

$0.09 \quad 12.64 \quad 1.07$

0.1

0.

0.1

$\begin{array}{lll} & 12.39 & 0.83\end{array}$

$\begin{array}{lll}0.14 & 12.57 & 0.88\end{array}$

$\begin{array}{lll}0.13 & 12.68 & 0.71\end{array}$

$0.71 \quad 0.06$

0.10

MgO

$\mathrm{CaO}$

$\mathrm{Na}_{2} \mathrm{O} \quad \mathrm{K}_{2} \mathrm{O}$

Cl Total

Sample

ID

$\begin{array}{lllll}\text { Core } & 77.89 & 0.11 & 12.62 & 0.91\end{array}$

$\begin{array}{lllll}\text { Core } & 77.80 & 0.12 & 12.56 & 0.81\end{array}$

$0.12 \quad 12.43 \quad 0.98$

0.05

$0.11 \quad 0.72$

$\begin{array}{lll}4.41 & 3.45 & 0.28\end{array}$

4.11 Bt 2.6

0.09

$0.10 \quad 0.67$

$4.23 \quad 3.44 \quad 0.15$

3.68 Bt 2.7

$0.09 \quad 0.11 \quad 0.73$

$\begin{array}{lll}4.30 & 3.31 & 0.16\end{array}$

4.27 Bt 2.9

0.05

$0.12 \quad 0.72$

$\begin{array}{lll}4.23 & 3.36 & 0.16\end{array}$

3.72 Bt 2.10

0.05

$0.10 \quad 0.7$

3.9

$4.17 \quad 0.16$

4.27 Bt 2.12

$\begin{array}{lll}0.09 & 0.10 \quad 0.73\end{array}$

$\begin{array}{lll}4.49 & 3.47 & 0.14\end{array}$

2.23 Bt_2.13

0.05

0.110 .76

$\begin{array}{lll}4.32 & 3.37 & 0.16\end{array}$

2.76 Bt_2.14

$\begin{array}{lll}0.08 & 0.12 & 0.7\end{array}$

$\begin{array}{lll}4.23 & 3.36 & 0.14\end{array}$

2.98 Bt_2.15

0.06

$0.12 \quad 0.72$

4.3

$0.14 \quad 3.25$ Bt 2.18

$0.08 \quad 0.14 \quad 0.72$

$\begin{array}{lll}4.04 & 3.37 & 0.13\end{array}$

4.86 Bt_2.19

$0.07 \quad 0.10 \quad 0.68$

4.20

3.29

0.13

2.62 Bt 2.20

$0.14 \quad 12.55 \quad 0.82$

0.08

$0.10 \quad 0.7$

$\begin{array}{lll}4.26 & 3.50 & 0.14\end{array}$

3.32 Bt 3.1

$\begin{array}{llll}12.55 & 0.82 & 0.10\end{array}$

$\begin{array}{lll}0.06 & 0.10 \quad 0.72\end{array}$

4.1

3.67

0.15

3.06 Bt 3.2

$\begin{array}{lll}0.06 & 0.15 & 0.69\end{array}$

$\begin{array}{lllll}4.36 & 3.25 & 0.15 & 0.57 & \text { Bt_3.3 }\end{array}$

$\begin{array}{lll}0.14 & 12.59 & 0.87\end{array}$

0.07

$0.13 \quad 0.70$

$\begin{array}{lllll}4.23 & 3.41 & 0.19 & 1.58 & \text { Bt_3.4 }\end{array}$

$\begin{array}{llll}0.11 & 12.37 & 0.93 & 0.05\end{array}$

$0.13 \quad 0.75$

4.24

$24 \quad 3.30 \quad 0.16$

36 Bt 3.5

$\begin{array}{lll}0.11 & 12.51 & 0.83\end{array}$

$\begin{array}{lll}0.06 & 0.14 & 0.73\end{array}$

$\begin{array}{lll}4.27 & 3.53 & 0.19\end{array}$

2.97 Bt 3.6

$0.11-12.46=0.79$

0.10

$0.11 \quad 0.72$

0.72

4.

$\begin{array}{lllll}.11 & 3.33 & 0.18 & 3.50 & \text { Bt_3.7 }\end{array}$

$\begin{array}{lllll}\text { Core } & 78.03 & 0.11 & 12.45 & 0.89\end{array}$

$0.12-12.57-0.92$

$\begin{array}{lll}0.06 & 0.10 & 0.72\end{array}$

$\begin{array}{lll}4.34 & 3.44 & 0.14\end{array}$

2.38 Bt_3.8

$\begin{array}{llll}0.12 & 12.57 & 0.92 & 0.09\end{array}$

$0.10 \quad 0.75$

$0.75 \quad 4.45$

$\begin{array}{lllll}4.36 & 3.36 & 0.22 & 4.53 & \text { Bt_3.11 }\end{array}$

$\begin{array}{lllll}4.33 & 3.38 & 0.16 & 3.64 & \text { Bt } 3.14\end{array}$

$\begin{array}{lllll}4.32 & 3.49 & 0.13 & 2.99 & \text { Bt_3.15 }\end{array}$

$\begin{array}{lllll}4.45 & 3.39 & 0.14 & 0.29 & \text { Bt } 3.16\end{array}$

$\begin{array}{lllll}.14 & 3.51 & 0.14 & 4.99 & \text { Bt_3.17 }\end{array}$

$\begin{array}{lllll}.45 & 3.43 & 0.16 & 1.77 & \text { Bt_3.18 }\end{array}$

0.1

4

\title{
$12.51-1.00 \quad 0.08$
}

$\begin{array}{lllll}4.44 & 3.23 & 0.19 & 3.56 & \text { Bt_3.19 }\end{array}$

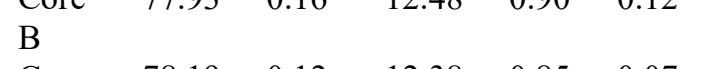

0.12

12.48
12.48

$\begin{array}{ll}1.00 & 0.08 \\ 0.90 \quad 0.12\end{array}$

0.12

0.86

4.08

$\begin{array}{llll}3.17 & 0.14 & 3.58 & \text { Bt_9.6 }\end{array}$

$\begin{array}{llll}3.22 & 0.15 & 3.45 & \text { Bt } 99.7\end{array}$

$\begin{array}{lllll}25 & 3.27 & 0.15 & 4.35 & \text { Bt_9.8 }\end{array}$

$\begin{array}{llllllllll}12.38 & 0.85 & 0.07 & 0.12 & 0.77 & 4.16 & 3.35 & 0.15 & 5.09 & \text { Bt_9.9 }\end{array}$ 
Tephra Bore-

Name

$\mathrm{SiO}$

$\mathrm{TiO}_{2}$

$\mathrm{Al}_{2} \mathrm{O}_{3}$

FeO

MnO

MgO

$\mathrm{CaO}$

$\mathrm{Na}_{2} \mathrm{O}$

$\mathrm{K}_{2} \mathrm{O}$

Total

Sample

Wh

Core $77.99 \quad 0$.

$\begin{array}{lll}12.37 & 1.07 & 0.04\end{array}$

0.13

$0.85 \quad 4.04$

$\begin{array}{lll}3.38 & 0.14 & 3.65\end{array}$

Wh B

$12.70 \quad 0.98 \quad 0.11$

0.12

0.91

1.60

3.48

$0.15 \quad 4.82$

Bt_9.10

Wh

B

12.71

$0.97 \quad 0.09$

$0.15 \quad 0.90$

B

$80.79 \quad 0.1$

$\begin{array}{lll}12.54 & 0.84 & 0.05\end{array}$

0.1

0.8

1.0

3.2

0.

5.58

Wh

B

Wh

$\begin{array}{llllll}\text { Core } & 78.07 & 0.13 & 12.44 & 0.98 & 0.06\end{array}$

0.13

0.86

4.26

3.28

$0.13 \quad 5.30$

Wh B

$12.44-0.98-0.06$

0.12

Core $\quad 78.27 \quad 0.14$

B

$\begin{array}{lll}12.42 & 0.86 & 0.06\end{array}$

0.12

$0.81 \quad 3.96$

$3.26 \quad 0.15 \quad 3.56$

Bt 9.17

Wh

$\begin{array}{llllll}\text { Core } & 77.92 & 0.14 & 12.44 & 1.02 & 0.05\end{array}$

0.120 .77

$.77 \quad 4.07$

3.35

$0.11 \quad 3.35$

Bt_9.18 B

Wh

$\begin{array}{llllll}\text { Core } & 77.81 & 0.15 & 12.28 & 1.01 & 0.08\end{array}$

0.11

$0.79 \quad 4.42$

$3.46 \quad 0.20 \quad 5.13$

Bt_9.19

A

$\mathrm{Rr}$

Core

$77.45 \quad 0.27$

1286

$1.17 \quad 0.07$

0.20

1.1

3.59 A

$3.33 \quad 1.32 \quad 0.07$

0.26

Core $\quad 76.68 \quad 0.25$

13.33

A

$12.90 \quad 1.22 \quad 0.10$

0.23

1.27

4.01

3.2

$0.14 \quad 4.78 \quad$ At_4_2

Core $\quad 77.02 \quad 0.25$

A

$12.86 \quad 1.22 \quad 0.04$

0.23

$1.22 \quad 4.09$

2.81

$0.16 \quad 2.20$

At_4_3

Core $\quad 77.16 \quad 0.23$

A

$\begin{array}{llll}12.87 & 1.24 & 0.12 & 0.20\end{array}$

1.21

4.04

2.96

$0.14 \quad 4.55 \quad$ At_4_4

Core $\quad 77.19 \quad 0.25$

A

$\begin{array}{lll}12.97 & 1.22 & 0.11\end{array}$

0.2

$1.16 \quad 3.88$

3.02

$0.13 \quad 3.91$

At_ 4 _5

Core $\quad 77.03 \quad 0.24$

A

$\begin{array}{lll}12.87 & 133 \quad 0.08\end{array}$

0.2

1.2

$26 \quad 3.98$

3.09

$0.15 \quad 3.69 \quad$ At_4_6

$\mathrm{Rr}$

$\mathrm{Rr}$

A

$12.82-1.23 \quad 0.03$

0.24

1.

$19 \quad 3.70$

2.98

$0.15 \quad 4.62$

At 47

Core $\quad 77.34 \quad 0.26$

$\begin{array}{lll}\text { A } & & \\ \text { Core } & 77.20 \quad 0.27\end{array}$

$\begin{array}{lll}12.82 & 1.23 & 0.03 \\ 12.82 & 1.25 & 0.08\end{array}$

0.27

$\begin{array}{lll}\text { A } & & \\ \text { Core } & 76.24 & 0.28\end{array}$

13.23

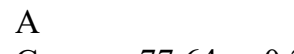

Core $\quad 77.64 \quad 0.20$

$13.23 \quad 1.36$

$36 \quad 0.09$

0.2

$\begin{array}{lll}12.75 & 1.00 & 0.07\end{array}$

0.

$\begin{array}{lll}\text { A } & & \\ \text { Core } & 76.87 & 0.28\end{array}$

A

$\begin{array}{lll}12.93 & 1.25 & 0.07\end{array}$

0.2

Core $\quad 77.10 \quad 0.30$

$\begin{array}{lll}12.96 & 1.23 \quad 0.03\end{array}$

0.26

1.3

$1.22 \quad 3.85$

2.91

$2.91 \quad 0.13 \quad 4.66$

At_4

$1 \overline{1}$

At_ 4

$1 \overline{3}$

At_4

$1 \overline{4}$

At_ 4

$1 \overline{5}$

At_4

$1 \overline{8}$

At_ 4

$\mathrm{Rr}$

\section{B}

Rw

Core $\quad 77.99 \quad 0.1$ B

$\begin{array}{lll}12.42 & 0.91 \quad 0.03\end{array}$

$0.14 \quad 0.83$

4.1

3.37

Core $\quad 77.69 \quad 0.09$

$$
\text { B }
$$

$\begin{array}{lll}12.44 & 0.88 & 0.08\end{array}$

0.07

$0.74 \quad 3.95$

.95

$4.07 \quad 0.18 \quad 3.23$

Bt_13.2

Rw

Core $\quad 78.04 \quad 0.1$

$12.50 \quad 1.00 \quad 0.05$

0.11

$0.84 \quad 4.04$

$\begin{array}{llll}3.31 & 0.15 & 4.53 & \text { Bt_13.3 }\end{array}$

$\begin{array}{lll}\text { B } & & \\ \text { Core } & 77.98 \quad 0.13\end{array}$

Rw

Rw

$$
\text { B }
$$

$12.49 \quad 0.93 \quad 0.04$

0.13

0.81

4.26

$\begin{array}{llll}3.22 & 0.14 & 3.27 & \text { Bt_13.4 }\end{array}$

Core $\quad 77.94 \quad 0.12$

$\begin{array}{lll}12.48 & 0.97 & 0.05\end{array}$

$0.11 \quad 0.84$

$\begin{array}{lllll}4.13 & 3.36 & 0.16 & 3.86 & \text { Bt_13.5 }\end{array}$

B

Core $\quad 77.85 \quad 0.12$

$$
\text { B }
$$

$\begin{array}{lll}12.42 & 1.03 & 0.07\end{array}$

0.1

Core $78.18 \quad 0.10$

$\begin{array}{lll}12.35 & 0.90 & 0.07\end{array}$

0.07

0.84

4.

4.17

$\begin{array}{llll}3.35 & 0.15 & 3.48 & \text { Bt_13.6 }\end{array}$

vRw

Rw

B

$\begin{array}{llllll}\text { Core } & 78.01 & 0.14 & 12.45 & 0.90 & 0.05\end{array}$

B

$12.41 \quad 1.01 \quad 0.08$

0.1

$0.65 \quad 3.85$

$\begin{array}{llll}3.82 & 0.16 & 3.91 & \text { Bt_13.7 }\end{array}$

Core $\quad 77.94 \quad 0.15$

$\begin{array}{lllllll}0.12 & 0.86 & 4.09 & 3.36 & 0.17 & 4.03 & \text { Bt_13.9 }\end{array}$ 
Tephra Bore-

Name

$\mathrm{SiO}_{2}$

$\mathrm{TiO}_{2}$

$\mathrm{Al}_{2} \mathrm{O}_{3}$

FeO

MnO

MgO

$\mathrm{CaO}$

$\mathrm{Na}_{2} \mathrm{O}$

$\mathrm{K}_{2} \mathrm{O}$

Total

Sample

Rw

B

$\begin{array}{lll}12.52 & 0.95 & 0.05\end{array}$

0.14

0.84

3.96

3.30

$0.14 \quad 4.52$

Bt_13.1

Rw B

$12.54 \quad 0.91 \quad 0.08$

0.12

0.79

4.19

3.20

Bt 13.1

Rw

B

$12.51 \quad 1.03 \quad 0.04$

0.1

0.83

4.03

$3.36 \quad 0.15 \quad 4.20$

Bt_13.1 B

$12.62 \quad 0.82 \quad 0.06$

0.05

$0.72 \quad 3.86$

$\begin{array}{lll}4.14 & 0.17 \quad 4.01\end{array}$

Bt 13.1

Rw

Core

12.64

$0.79 \quad 0.05$

0.0

0.7

$71 \quad 3.97$

97

$\begin{array}{lll}4.18 & 0.16 \quad 4.67\end{array}$

Bt 13.1

$\begin{array}{lll}\text { Core } & 77.52 & 0 \\ \mathrm{~B} & \end{array}$

$\begin{array}{lll}12.49 & 0.95 & 0.02\end{array}$

0.1

0.

B $\quad 77.98 \quad 0.12$

$\begin{array}{lll}12.47 & 0.98 \quad 0.08\end{array}$

0.12

0.82

A

Ok

Core

$78.18 \quad 0.14$

12.48

$0.93 \quad 0.09$

$\begin{array}{llllll}0.14 & 0.79 & 3.64 & 3.61 & 0.16 & 4.99\end{array}$

At_5_co A $\quad 77.00 \quad 0.22$

$\mathrm{Ok}$

Ok

Core $\quad 77.00 \quad 0.22$

A

13

13

$14 \quad 1.21 \quad 0.10$

$0.21 \quad 1.00$

$1.00 \quad 4.09$

$4.09 \quad 3.02$

$\begin{array}{lll}3.02 & 0.19 \quad 4.61\end{array}$

rr_- 1

At_5_co

rr 3

At_5_co $\begin{array}{lll}\text { Core } & 77.88 & 0.18 \\ \text { A } & \end{array}$

$12.48 \quad 0.92 \quad 0.06$

0.14

$0.82 \quad 3.6$

$3.92 \quad 0.15 \quad 4.28$

rr $\overline{4}$

At 5 _co

Ok

Ok

Core $\quad 78.17 \quad 0.17$

$\begin{array}{lll}12.48 & 0.85 & 0.04\end{array}$

$\begin{array}{lll}0.12 & 0.77 \quad 3.91\end{array}$

$\begin{array}{lll}3.50 & 0.17 \quad 4.69\end{array}$

rr

$\begin{array}{lll}\text { A } & & \\ \text { Core } & 77.87 & 0.15\end{array}$

$\begin{array}{lll}12.59 & 0.84 & 0.06\end{array}$

0.13

$\begin{array}{lllll}0.79 & 3.94 & 3.63 & 0.16 & 4.68\end{array}$

At_5_co A

$12.54 \quad 0.85 \quad 0.10$

0.1

0.79

39.79

A

$\begin{array}{lll}12.52 & 0.85 & 0.07\end{array}$

0.1

A $\quad 78.08 \quad 0.13$

Ok

Ok

A

$\begin{array}{lll}12.52 & 0.95 & 0.08\end{array}$

0.13

0.75

$.75 \quad 3.83$

3.60

$\begin{array}{lll}.60 & 0.14 & 5.33\end{array}$

At̄5_co

rr 7 -

At_5_co

rr 10

At 5 co

rr_11

At_5_co Core $77.98 \quad 0.15$

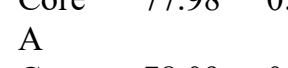

Ok

Ok

Core $\quad 78.09 \quad 0.12$

$$
\text { A }
$$

$\begin{array}{lll}12.61 & 0.89 & 0.03\end{array}$

0.1

$0.80 \quad 3.7$

$3.58 \quad 0.16 \quad 4.46$

rr 13

At̄ 5_co

$12.44 \quad 0.86 \quad 0.05$

0.1

$\begin{array}{lllll}0.79 & 3.89 & 3.51 & 0.15 & 4.59\end{array}$

At_5_co Core $78.09 \quad 0.13$ $\begin{array}{lll}\text { A } & \\ \text { Core } & 77.95 \quad 0.13\end{array}$

Ok

Ok

B

$\begin{array}{lll}12.62 & 0.88 & 0.09\end{array}$

0.1

$0.73 \quad 3.60$

$3.99 \quad 0.14 \quad 4.11$

At_5_co

$12.47 \quad 0.94 \quad 0.10$

0.10

$\begin{array}{ll}0.76 \quad 3.61 \\ 0.76 & 4.01\end{array}$

$\begin{array}{lll}3.68 & 0.19 \quad 4.92\end{array}$

Core $77.24 \quad 0.21$

$12.66 \quad 1.24 \quad 0.06$

0.1

$0.84-3.94$

3.5

$0.16 \quad 4.26$

rr_18

Ok

Core $\quad 77.94 \quad 0.1$

B

$12.51 \quad 1.01 \quad 0.02$

0.06

0.84

Ok

Ok

Core $\quad 77.58 \quad 0.17$

$$
\text { B }
$$

$\begin{array}{lll}12.61 & 0.87 & 0.05\end{array}$

0.1

$\begin{array}{llllll}\text { Core } & 78.14 & 0.21 & 12.28 & 1.01 & 0.06\end{array}$

0.170 .83

$$
\text { B }
$$

$\begin{array}{lll}13.17 & 1.24 & 0.07\end{array}$

$0.19 \quad 1.08$

$$
\text { B }
$$

Ok

$\mathrm{Ok}$

$\begin{array}{llllll}\text { Core } & 78.13 & 0.15 & 12.55 & 0.9 & 0.03\end{array}$

0.1

$$
\text { B }
$$

Core $\quad 78.04 \quad 0.15$

B

Ok

Core $\quad 78.04 \quad 0.14$

B

$12.37 \quad 1.01 \quad 0.02$

0.1

0.7

$0.72 \quad 3.95$

$0.77 \quad 3.93$

$\begin{array}{lll}12.6 & 0.93 \quad 0.05\end{array}$

$\begin{array}{lll}0.14 & 0.75 \quad 3.73\end{array}$

3.7

$0.11 \quad 4.73$

Bt15 3

Ok

Core $\quad 78.03 \quad 0.1$

$\begin{array}{lll}\text { B } & & \\ \text { Core } & 77.85 & 0.17\end{array}$

$\begin{array}{lll}12.52 & 0.92 & 0.04\end{array}$

$\begin{array}{lll}0.13 & 0.69 \quad 3.6\end{array}$

$\begin{array}{lll}12.63 & 0.93 & 0.07\end{array}$

$\begin{array}{lll}0.14 & 0.74 \quad 3.9\end{array}$

$\mathrm{Ok}$ Core $\quad 77.85 \quad 0$. 
Tephra Bore-

$\mathrm{SiO}_{2}$

$\mathrm{TiO}_{2}$

$\mathrm{Al}_{2} \mathrm{O}_{3} \quad \mathrm{FeO} \quad \mathrm{MnO}$

MgO

$\mathrm{CaO}$

$\mathrm{Na}_{2} \mathrm{O}$

$\mathrm{K}_{2} \mathrm{O}$

Total

Sample

$\mathrm{Kw}$

B

$12.45 \quad 1.25 \quad 0.01$

0.03

$0.46 \quad 2.41$

4.94

$0.11 \quad 1.56$

$\mathrm{Kw}$ B

$12.51 \quad 1.16 \quad 0.01$

0.03

0.45

1.59

5.01

$0.10 \quad 1.12$

Bt_19.1

Kw

B

$12.46 \quad 1.08 \quad 0.02$

$0.11 \quad 0.99$

4.16

3.17

$0.19 \quad 4.71$

$\mathrm{Kw}$ B

$12.50 \quad 1.16 \quad 0.04$

0.13

1.03

B

Kw

$\begin{array}{llllll}\text { Core } & 78.05 & 0.13 & 12.51 & 1.19 & 0.03\end{array}$

0.12

0.95

3.99

3.01

$0.20 \quad 5.72$

Bt_19.5 B

$\mathrm{Kw}$

$\mathrm{Kw}$

Core $77.88 \quad 0$

B

Core $77.90 \quad 0.13$

$12.48 \quad 1.08 \quad 0.04$

$0.12 \quad 0.94 \quad 4.20$

3.

$0.20 \quad 5.30$

Bt 19.6

$\mathrm{Kw}$

$\mathrm{Kw}$

B

$12.50 \quad 1.14 \quad 0.06$

0.11

0.94

4.02

$\begin{array}{lll}3.14 & 0.19 & 5.79\end{array}$

Bt_19.7 Core $\quad 77.30 \quad 0$

B

$12.86 \quad 1.25 \quad 0.05$

0.1

1.05

4.10

3.2

$0.21 \quad 4.17$

Bt 19.8

Bt_19.1

Core $77.38 \quad 0$

$\begin{array}{lll}12.59 & 1.23 \quad 0.08\end{array}$

0.13

0.98

4.4

3.11

$0.20 \quad 4.49$

0

$\mathrm{Kw}$

$\mathrm{Kw}$

Core $\quad 79.99 \quad 0$.

B

$12.84 \quad 1.34 \quad 0.04$

0.1

1.04

Core $77.64 \quad 0$

$\begin{array}{lll}12.53 & 1.26 & 0.04\end{array}$

0.1

1.0

$\mathrm{Kw}$

Kw

Core $\quad 77.78 \quad 0$

B

$\begin{array}{lll}12.51 & 1.14 & 0.07\end{array}$

0.1

1.04

Core $77.75 \quad 0$

$\begin{array}{lll}12.52 & 1.19 & 0.04\end{array}$

0.1

1.56

3.05

$0.19 \quad 2.67$

Bt_19.1

3

Bt 19.1

4
Bt 19.1

Bt_19.1

Bt_19.1

Bt 19.2

Ok $\quad$ B

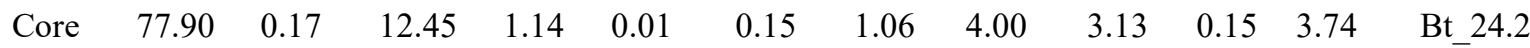

Ok

B

Core $\quad 77.88 \quad 0$

Ok

B

Core $\quad 77.52 \quad 0.17$

0.17

$12.51 \quad 1.14 \quad 0.00$

$0.12 \quad 1.03$

4.14

$\begin{array}{lll}3.01 & 0.16 \quad 2.10\end{array}$

Bt_24.3

Ok

B

$12.54 \quad 1.29 \quad 0.08$

$0.13 \quad 1.09 \quad 4.05$

$3.12 \quad 0.13 \quad 2.12 \quad$ Bt_24.4

Core $\quad 77.55 \quad 0.1$

$\begin{array}{lll}12.59 & 1.23 & 0.04\end{array}$

$0.15 \quad 1.10 \quad 4.1$

$3.120 .16 \quad 3.83$

Bt_24.5

Ok

$\mathrm{Ok}$

Core $77.87 \quad 0.14$

$12.49 \quad 1.10 \quad 0.06$

0.13

B

Core $77.81 \quad 0.15$

Ok

Ok

B

$\begin{array}{lll}12.43 & 1.05 & 0.09\end{array}$

$0.10 \quad 0.95$

$0.99 \quad 3.86$

$\begin{array}{lll}3.36 & 0.14 & 2.97\end{array}$

Bt 24.6

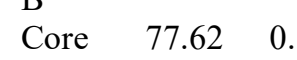

$12.40-1.15=0.06$

$0.15 \quad 1.10$

$95 \quad 3.98$

$3.45 \quad 0.15 \quad 3.44$

Bt_24.7

B

$\begin{array}{llllll}\text { Core } & 77.88 & 0.14 & 12.56 & 1.21 & 0.07\end{array}$

$0.15 \quad 1$.

4.13

3.26

$0.14 \quad 1.79$

Bt_24.8

$\mathrm{Ok}$

$\mathrm{Ok}$

Core $77.72 \quad 0$

$12.38-1.30=0.03$

$0.15 \quad 1.11$

103

3.83

$3.07 \quad 0.15 \quad 3.73$

Bt_24.9

Bt 24.1

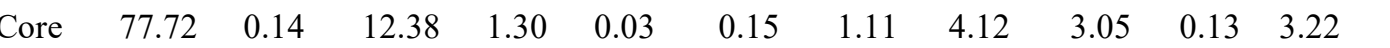

Core $\quad 77.96 \quad 0.13$

$12.45 \quad 1.23 \quad 0.10$

0.13

1.10

3.84

0

Bt_24.1

Ok

Core $77.77 \quad 0.14$

$12.40-1.10 \quad 0.06$

0.14

1.

11

3.84

$3.05 \quad 0.14 \quad 3.16$

Bt 24.1

$\mathrm{Ok}$

B

$$
\begin{array}{llllll}
\text { Core } & 77.80 & 0.12 & 12.45 & 1.11 & 0.04
\end{array}
$$

0.15

0.98

98

4.10

3.49

0.14

Bt_24.1

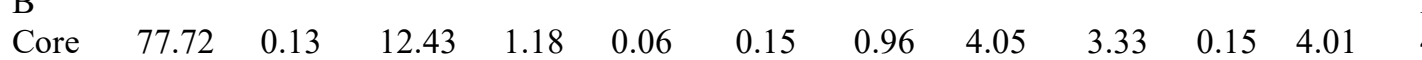

$\begin{array}{lllllllllll}\text { B } & & & & & & & & & & \end{array}$

$\begin{array}{llllllllllll}\text { B } & & & & & & & & & & & \\ \text { Core } & 77.66 & 0.13 & 12.39 & 1.08 & 0.06 & 0.14 & 0.99 & 4.14 & 3.42 & 0.14 & 2.14\end{array}$

$\begin{array}{llllll}\mathrm{B} & & & & & \end{array}$

$\begin{array}{lllllllllllll}\text { Core } & 77.53 & 0.12 & 12.54 & 1.24 & 0.11 & 0.14 & 1.09 & 4.08 & 3.14 & 0.16 & 2.68 & 7\end{array}$

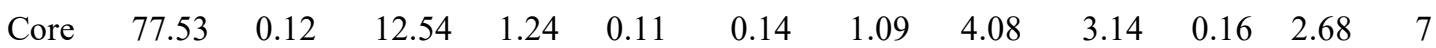

Bt_24.1

4

Bt_24.1

Bt 24.1

Bt 24.1 
Tephra Bore-

SiO

$\mathrm{TiO}_{2}$

$\mathrm{Al}_{2} \mathrm{O}_{3}$

FeO

MnO

MgO

$\mathrm{CaO}$

$\mathrm{Na}_{2} \mathrm{O}$

$\mathrm{K}_{2} \mathrm{O}$

Cl

Total

Sample

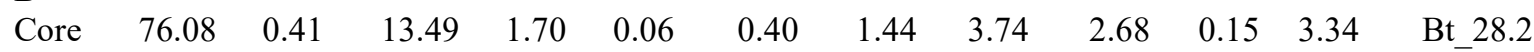

$\mathrm{Ha}$

B

Core $\quad 73.17 \quad 0.54$

B

$14.45 \quad 2.54 \quad 0.08$

0.63

$2.12 \quad 4.05$

$2.42 \quad 0.13 \quad 1.36$

Bt_28.3

$\mathrm{Ha}$

Core $75.72 \quad 0.41$

$13.46 \quad 1.74 \quad 0.12$

$\begin{array}{llllll}0.44 & 1.54 & 3.90 & 2.66 & 0.14 & 1.21\end{array}$

Bt 28.4

$\mathrm{Ha}$

$\mathrm{Ha}$

Core $\quad 75.92 \quad 0.37$

B

$13.52 \quad 1.73 \quad 0.06$

0.44

$1.52 \quad 3.79$

2.63

$2.63 \quad 0.13 \quad 1.84$

Bt 28.5

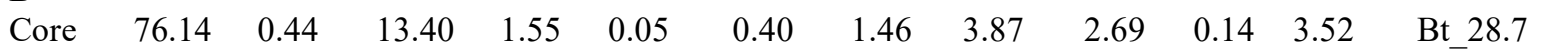

$\mathrm{Ha}$

$\mathrm{Ha}$

Core $\quad 75.98 \quad 0.40$

$\begin{array}{lll}\text { B } & & \\ \text { Core } & 76.09 \quad 0\end{array}$

$\begin{array}{lll}13.51 & 1.65 & 0.07\end{array}$

0.43

$1.48 \quad 3.79$

$\begin{array}{lll}2.69 & 0.14 & 3.54\end{array}$

Bt_28.8

$\mathrm{Ha}$

$\begin{array}{lll}13.55 & 1.57 & 0.07\end{array}$

$0.34 \quad 1.42 \quad 3.83$

$\begin{array}{lll}2.68 & 0.12 & 1.89\end{array}$

Bt 28.9

Core $\quad 73.43 \quad 0.56$

B

$\begin{array}{lll}14.23 & 2.39 & 0.10\end{array}$

0.62

2.05

4.15

2.47

Bt 28.1

Core $\quad 76.13 \quad 0.39$

$\begin{array}{lll}13.55 & 1.58 & 0.07\end{array}$

0.4

1.4

3.79

Core $\quad 76.72 \quad 0.3$

B

$\begin{array}{lll}13.25 & 1.45 \quad 0.08\end{array}$

0.3

1.

Core $\quad 76.07 \quad 0.38$

$\begin{array}{lll}13.39 & 1.60 & 0.07\end{array}$

0.4

1.4

B

Core $\quad 75.90 \quad 0.4$

B

$13.47 \quad 1.88 \quad 0.06$

0.4

1.51

Core $\quad 76.87 \quad 0.31$

$\begin{array}{lll}13.32 & 1.42 & 0.05\end{array}$

0.3

1.3

B

Core $\quad 74.60 \quad 0.43$

B

13.71

$2.15 \quad 0.08$

0.5

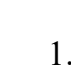

Core $74.83 \quad 0.4$

$\begin{array}{lll}13.92 & 2.03 & 0.08\end{array}$

0.50

1.75

$\begin{array}{lll}\text { B } & & \\ \text { Core } & 75.49 & 0.4\end{array}$

$\begin{array}{lll}14.05 & 1.87 & 0.07\end{array}$

0.48

1.48

Mk B

Mk

$\mathrm{Mk}$

$\mathrm{Mk}$

$\mathrm{Mk}$

$\mathrm{Mk}$

$\mathrm{Mk}$

$\mathrm{Mk}$

Mk

$\mathrm{Mk}$

$\mathrm{Mk}$

$\mathrm{Mk}$

$\mathrm{Mk}$

Core $\quad 73.60 \quad 0.55$

$$
\text { B }
$$

Core $\quad 73.90 \quad 0.53$

$14.35 \quad 2.22$

0.14

0.60

1.93

4.17

2.65

$0.13 \quad 0.79$

0

Bt_28.1

Bt 28.1

2

Bt 28.1

Bt 28.1

Bt 28.1

5

Bt_28.1

Bt 28.1

$$
\text { B }
$$

$\begin{array}{lll}14.23 & 2.19 & 0.10\end{array}$

$0.62 \quad 1.88$

4.13

Core $\quad 73.79 \quad 0.55$

B

$14.04 \quad 2.26 \quad 0.11$

0.63

$1.91 \quad 4.35$

Core $\quad 73.68 \quad 0.55$

14.28

$2.20 \quad 0.11$

0.57

1.87

4.45

B

Core $76.41 \quad 0.3$

$\begin{array}{lll}13.34 & 1.39 & 0.07\end{array}$

0.37

1.29

4.14

$\begin{array}{lll}\text { Core } & 76.58 & 0.34 \\ \text { B } & & \end{array}$

$\begin{array}{lll}13.35 & 1.42 & 0.05\end{array}$

$0.34 \quad 1.34$

4.12

Core $\quad 76.89 \quad 0.28$

$\begin{array}{lll}13.36 & 1.30 & 0.08\end{array}$

0.3

1.2

B

Core $\quad 75.29 \quad 0.4$

$\begin{array}{lll}13.95 & 1.71 & 0.07\end{array}$

0.45

1.45

3.87

$\begin{array}{lll}2.45 & 0.17 & 0.03\end{array}$

B

$\begin{array}{lll}\text { Core } & 76.70 \quad 0.27 & 13\end{array}$

$13.37 \quad 1.30 \quad 0.07$

0.29

$1.31 \quad 3.99$

$2.64 \quad 0.15 \quad 2.55$

Bt_29.8

Core $\quad 74.35 \quad 0$.

$13.99 \quad 2.23 \quad 0.06$

0.56

1.70

4.13

4.13

$0.15 \quad 1.14$

Bt 29.9

Bt_29.1

$\begin{array}{lll}\text { B } & & \\ \text { Core } & 76.52 & 0.29\end{array}$

$\begin{array}{lll}\text { Core } & 76.52 & 0 \\ \text { B }\end{array}$

Core $\quad 75.72 \quad 0.36$

13.2

$1.45 \quad 0.04$

0.3

1.3

$.34 \quad 4.2$

4.22

$2.70 \quad 0.14 \quad 3.08$

Bt 29.1

1

Bt_29.1

$\begin{array}{lll}\text { B } & & \\ \text { Core } & 76.73 \quad 0.33\end{array}$

Mk

$\begin{array}{lll}\text { Core } & 76.73 & 0.33 \\ \text { B } & & \end{array}$

$\begin{array}{lll}13.25 & 1.43 \quad 0.06\end{array}$

0.3

1.

$1.28 \quad 3.95$

3.95

$2.64 \quad 0.14 \quad 2.14$

Core $\quad 76.73 \quad 0.3$

$\begin{array}{lll}13.38 & 1.49 & 0.05\end{array}$

$\begin{array}{lll}0.31 & 1.32 \quad 3.7\end{array}$

2.63

$0.17 \quad 2.10 \quad 5$

Bt_29.1

3

Bt_29.1

Bt_29.1 


\begin{tabular}{|c|c|c|c|c|c|c|c|c|c|c|c|c|c|}
\hline $\begin{array}{l}\text { Tephra } \\
\text { Name } \\
\text { Mk }\end{array}$ & $\begin{array}{l}\text { Bore- } \\
\text { hole } \\
\text { B }\end{array}$ & $\mathrm{SiO}_{2}$ & $\mathrm{TiO}_{2}$ & $\mathrm{Al}_{2} \mathrm{O}_{3}$ & $\mathrm{FeO}$ & MnO & MgO & $\mathrm{CaO}$ & $\mathrm{Na}_{2} \mathrm{O}$ & $\mathrm{K}_{2} \mathrm{O}$ & Cl & Total & $\begin{array}{l}\text { Sample } \\
\text { ID } \\
\text { Bt_29.1 }\end{array}$ \\
\hline & Core & 73.83 & 0.51 & 14.12 & 2.35 & 0.08 & 0.60 & 1.90 & 4.16 & 2.44 & 0.18 & 1.10 & $6^{-}$ \\
\hline Mk & B & & & & & & & & & & & & Bt_29.1 \\
\hline & Core & 76.54 & 0.33 & 13.48 & 1.33 & 0.08 & 0.33 & 1.30 & 3.98 & 2.63 & 0.17 & 2.00 & $7^{-}$ \\
\hline Mk & B & & & & & & & & & & & & Bt_29.1 \\
\hline Mk & $\begin{array}{l}\text { Core } \\
\text { B }\end{array}$ & 74.17 & 0.52 & 14.12 & 2.11 & 0.09 & 0.60 & 1.91 & 4.23 & 2.24 & 0.15 & 2.84 & $\begin{array}{l}9 \\
\text { Bt_29.2 }\end{array}$ \\
\hline $\mathrm{Mk}$ & $\begin{array}{l}\text { Core } \\
\text { B }\end{array}$ & 76.53 & 0.28 & 13.35 & 1.53 & 0.06 & 0.30 & 1.33 & 3.98 & 2.63 & 0.17 & 3.11 & $0^{-}$ \\
\hline Mk & $\begin{array}{l}\text { Core } \\
\text { A }\end{array}$ & 73.79 & 0.55 & 14.04 & 2.26 & 0.11 & 0.63 & 1.91 & 4.35 & 2.37 & 0.15 & 0.11 & Bt_29.3 \\
\hline Mk & $\begin{array}{l}\text { Core } \\
\text { A }\end{array}$ & 76.12 & 0.24 & 13.23 & 1.36 & 0.11 & 0.31 & 1.35 & 4.68 & 2.62 & 0.19 & 2.21 & At_7.1 \\
\hline Mk & $\begin{array}{l}\text { Core } \\
\text { A }\end{array}$ & 77.81 & 0.14 & 12.57 & 1.07 & 0.06 & 0.13 & 0.89 & 3.54 & 3.78 & 0.14 & 1.77 & At_7.2 \\
\hline $\mathrm{Mk}$ & $\begin{array}{l}\text { Core } \\
\text { A }\end{array}$ & 77.70 & 0.17 & 12.52 & 1.07 & 0.06 & 0.15 & 0.99 & 3.28 & 4.06 & 0.16 & 3.26 & At_7.3 \\
\hline Mk & $\begin{array}{l}\text { Core } \\
\text { A }\end{array}$ & 77.87 & 0.15 & 12.39 & 1.04 & 0.05 & 0.12 & 0.92 & 3.63 & 3.84 & 0.17 & 3.71 & At_7.4 \\
\hline Mk & $\begin{array}{l}\text { Core } \\
\text { A }\end{array}$ & 76.42 & 0.33 & 13.24 & 1.32 & 0.07 & 0.26 & 1.35 & 4.38 & 2.63 & 0.17 & 2.47 & At_7.8 \\
\hline Mk & $\begin{array}{l}\text { Core } \\
\text { A }\end{array}$ & 76.34 & 0.31 & 13.35 & 1.33 & 0.11 & 0.28 & 1.33 & 4.29 & 2.65 & 0.16 & 3.08 & At_7.9 \\
\hline $\mathrm{Mk}$ & $\begin{array}{l}\text { Core } \\
\text { A }\end{array}$ & 73.39 & 0.53 & 14.22 & 2.21 & 0.13 & 0.55 & 1.98 & 4.68 & 2.32 & 0.19 & 0.06 & At_7.11 \\
\hline $\mathrm{Mk}$ & $\begin{array}{l}\text { Core } \\
\text { A }\end{array}$ & 77.83 & 0.17 & 12.62 & 1.28 & 0.05 & 0.12 & 1.01 & 4.11 & 2.83 & 0.18 & 5.84 & At_7.13 \\
\hline $\mathrm{Mk}$ & Core & 73.39 & 0.52 & 14.02 & 2.31 & 0.14 & 0.56 & 1.94 & 4.70 & 2.41 & 0.19 & 0.08 & At_7.15 \\
\hline Mk & $\begin{array}{l}\text { Core } \\
\text { A }\end{array}$ & 76.01 & 0.30 & 13.28 & 1.46 & 0.11 & 0.31 & 1.38 & 4.55 & 2.60 & 0.18 & 1.16 & At_7.16 \\
\hline & Core & 75.89 & 0.31 & 13.30 & 1.57 & 0.07 & 0.30 & 1.26 & 4.51 & 2.80 & 0.18 & 2.24 & At_7.18 \\
\hline Ta & $\begin{array}{l}\text { B } \\
\text { Core }\end{array}$ & 77.56 & 0.14 & 12.55 & 0.96 & 0.01 & 0.14 & 0.89 & 3.71 & 4.04 & 0.12 & 2.82 & Bt31.1 \\
\hline $\mathrm{Ta}$ & $\begin{array}{l}\text { B } \\
\text { Core }\end{array}$ & 78.48 & 0.14 & 12.28 & 1.00 & 0.02 & 0.14 & 0.92 & 3.00 & 4.00 & 0.15 & 1.77 & Bt31.2 \\
\hline Ta & B & & & & & & & & & & & & \\
\hline $\mathrm{Ta}$ & $\begin{array}{l}\text { Core } \\
\text { B }\end{array}$ & 78.62 & 0.15 & 12.22 & 0.19 & 0.00 & 0.01 & 0.64 & 3.71 & 4.47 & 0.04 & 1.43 & Bt31.3 \\
\hline $\mathrm{Ta}$ & $\begin{array}{l}\text { Core } \\
\text { B }\end{array}$ & 77.94 & 0.17 & 12.44 & 0.97 & 0.02 & 0.10 & 0.88 & 3.40 & 4.08 & 0.15 & 4.50 & Bt31.5 \\
\hline $\mathrm{Ta}$ & $\begin{array}{l}\text { Core } \\
\text { B }\end{array}$ & 77.77 & 0.13 & 12.57 & 1.00 & 0.01 & 0.12 & 0.88 & 3.36 & 4.14 & 0.15 & 4.36 & Bt31.6 \\
\hline $\mathrm{Ta}$ & $\begin{array}{l}\text { Core } \\
\text { B }\end{array}$ & 77.75 & 0.14 & 12.50 & 0.98 & 0.04 & 0.15 & 0.84 & 3.64 & 3.95 & 0.16 & 1.96 & Bt31.7 \\
\hline $\mathrm{Ta}$ & $\begin{array}{l}\text { Core } \\
\text { B }\end{array}$ & 77.75 & 0.12 & 12.50 & 0.94 & 0.03 & 0.14 & 0.87 & 3.78 & 3.88 & 0.16 & 3.49 & Bt31.8 \\
\hline Ta & $\begin{array}{l}\text { Core } \\
\text { B }\end{array}$ & 77.82 & 0.17 & 12.33 & 0.98 & 0.04 & 0.14 & 0.90 & 3.42 & 4.19 & 0.13 & 4.24 & Bt31.9 \\
\hline $\mathrm{Ta}$ & $\begin{array}{l}\text { Core } \\
\text { B }\end{array}$ & 77.81 & 0.14 & 12.52 & 0.93 & 0.01 & 0.15 & 0.90 & 3.43 & 4.12 & 0.12 & 5.15 & Bt31.11 \\
\hline $\mathrm{Ta}$ & $\begin{array}{l}\text { Core } \\
\text { B }\end{array}$ & 77.92 & 0.15 & 12.32 & 1.06 & 0.03 & 0.14 & 0.81 & 3.47 & 4.10 & 0.15 & 5.21 & Bt31.12 \\
\hline $\mathrm{Ta}$ & $\begin{array}{l}\text { Core } \\
\text { B }\end{array}$ & 78.00 & 0.14 & 12.33 & 0.99 & 0.06 & 0.15 & 0.90 & 3.43 & 4.00 & 0.13 & 3.55 & Bt31.14 \\
\hline $\mathrm{Ta}$ & $\begin{array}{l}\text { Core } \\
\text { B }\end{array}$ & 77.84 & 0.16 & 12.57 & 0.98 & 0.02 & 0.13 & 0.88 & 3.29 & 4.13 & 0.16 & 5.14 & Bt31.15 \\
\hline Ta & $\begin{array}{l}\text { Core } \\
\text { B }\end{array}$ & 77.71 & 0.18 & 12.50 & 1.03 & 0.04 & 0.12 & 0.87 & 3.54 & 4.01 & 0.12 & 2.84 & Bt31.18 \\
\hline & Core & 78.07 & 0.18 & 12.27 & 1.06 & 0.02 & 0.16 & 0.82 & 3.46 & 3.98 & 0.14 & 3.66 & Bt31.19 \\
\hline
\end{tabular}


$12.48 \quad 1.06 \quad 0.04$

$\begin{array}{llllll}0.14 & 0.94 & 3.52 & 3.92 & 0.13 & 4.77\end{array}$

Bt31.20

Re A

$\operatorname{Re}$

$\mathrm{Re}$

Re

$\operatorname{Re}$

$\operatorname{Re}$

$\operatorname{Re}$

$\operatorname{Re}$

$\operatorname{Re}$

$\operatorname{Re}$

$\operatorname{Re}$

$\operatorname{Re}$

$\operatorname{Re}$

$\operatorname{Re}$

Re

$\mathrm{Re}$

$\operatorname{Re}$

$\operatorname{Re}$

$\operatorname{Re}$

$\operatorname{Re}$

$\mathrm{Re}$

$\operatorname{Re}$

$\mathrm{Re}$

$\mathrm{Re}$

$\mathrm{Re}$

$\operatorname{Re}$

Re

$\operatorname{Re}$

Core A Core $78.10 \quad 0$ A Core $\quad 78.34 \quad 0$ A Core $\quad 78.09 \quad 0.1$ A Core $\quad 78.20 \quad 0.14$ A Core $\quad 78.42 \quad 0.14$ A Core $\quad 77.95 \quad 0.15$ A Core $\quad 78.28 \quad 0.16$ A Core $\quad 78.00 \quad 0.15$ A Core $\quad 78.20 \quad 0.1$ A Core $\quad 78.04 \quad 0.15$ A Core $\quad 77.94 \quad 0.12$

A Core $\quad 78.15 \quad 0.14$

0.12

A Core $\quad 77.75 \quad 0.13$ A Core $\quad 78.46 \quad 0.15$ A Core $\quad 77.92 \quad 0.13$ A Core $\quad 78.09 \quad 0.14$ A Core $\quad 77.87 \quad 0.14$ A Core $\quad 78.08 \quad 0.17$

$\begin{array}{llll}0.14 & 12.66 & 1.08 & 0.10\end{array}$

$\begin{array}{lll}12.66 & 1.08 & 0.10\end{array}$ A Core $\quad 78.08 \quad 0.15$ A Core $\quad 77.75 \quad 0.18$ A Core $\quad 78.14 \quad 0.18$ A Core $\quad 78.12 \quad 0.15$ A Core $\quad 77.87 \quad 0.16$ A Core $\quad 78.36 \quad 0.15$ A Core $\quad 77.73 \quad 0.15$ A Core $\quad 78.03 \quad 0.14$ A

$\begin{array}{llllll}\text { Core } & 77.76 & 0.15 & 12.70 & 0.98 & 0.10\end{array}$

$\begin{array}{lll}12.82 & 0.95 & 0.05\end{array}$

$12.64 \quad 0.84 \quad 0.03$

$\begin{array}{lll}12.62 & 0.97 & 0.09\end{array}$

$\begin{array}{lll}12.80 & 1.03 \quad 0.06\end{array}$

$\begin{array}{lll}12.76 & 0.87 & 0.06\end{array}$

$\begin{array}{lll}12.50 & 0.84 & 0.08\end{array}$

$\begin{array}{lll}12.68 & 0.93 & 0.03\end{array}$

$\begin{array}{lll}12.52 & 1.01 & 0.08\end{array}$

$12.71 \quad 0.94 \quad 0.05$

$\begin{array}{lll}12.71 & 0.89 & 0.05\end{array}$

$12.88 \quad 0.90 \quad 0.05$

$\begin{array}{lll}12.75 & 1.08 & 0.03\end{array}$

$\begin{array}{lll}12.70 & 0.96 \quad 0.06\end{array}$

$12.74 \quad 1.05 \quad 0.06$

$12.62 \quad 1.03 \quad 0.05$

$12.74 \quad 0.86 \quad 0.03$ 0.1

0.14

0.17

0.7

12.73

$\begin{array}{lll}12.58 & 0.84 & 0.07\end{array}$ 0.1

0.16

0.69

0.69

4.35

.35

3.13

3.13

0.

$0.20 \quad 3.49 \quad$ At_8.19

$\begin{array}{lll}12.45 & 0.98 & 0.06\end{array}$

$$
0.17
$$

0.79

4.07

$3.08 \quad 0.20 \quad 5.36$

At9.1

$\begin{array}{lll}12.62 & 0.80 & 0.10\end{array}$ 0.14

0.80

3.88

3.3

$0.19 \quad 4.41$

At9. 2

0.16

0.80

3.96

$\begin{array}{lll}3.28 & 0.22 \quad 5.77\end{array}$

At9.3

17

0.82

3.93

3.3

$0.21 \quad 3.15$

At9.4

$\begin{array}{lll}12.68 & 0.89 & 0.11\end{array}$

0.19

0.79

4.27

$\begin{array}{lll}3.36 & 0.20 & 3.72\end{array}$

At9.5

$\begin{array}{lll}12.68 & 0.96 & 0.10\end{array}$

$\begin{array}{lll}0.17 & 0.79 \quad 3.91\end{array}$

3.27

$0.19 \quad 5.07$

At9.6

$\begin{array}{lll}12.50 & 0.84 & 0.06\end{array}$

0.13

0.84

3.98

3.35

$\begin{array}{lll}3.62 & 0.24 & 5.87\end{array}$

At9.7

$\begin{array}{lll}12.64 & 0.80 & 0.13\end{array}$

0.1

0.8

$0.82 \quad 4.2$

4.24

3.

$12.60 \quad 0.92 \quad 0.11$

0.12

$0.80 \quad 4.09$

$\begin{array}{llll}3.34 & 0.19 & 4.01 & \text { At9.10 }\end{array}$

$\begin{array}{lll}0.16 & 0.85 & 4.30\end{array}$

$3.19 \quad 0.21 \quad 5.13$

At9.11

$\begin{array}{lll}\text { A } & & \\ \text { Core } & 78.28 & 0.14\end{array}$

$\begin{array}{lll}12.70 & 0.98 \quad 0.10\end{array}$

0.16

$0.81 \quad 4.08$ $\begin{array}{llll}2.99 & 0.21 & 4.28 & \text { At9.12 }\end{array}$

$\begin{array}{llll}3.05 & 0.19 & 5.03 & \text { At9.14 }\end{array}$ 
Tephra Bore-

$\mathrm{Al}_{2} \mathrm{O}_{3}$

FeO

MnO

MgO

$\mathrm{CaO}$

$\mathrm{Na}_{2} \mathrm{O}$

$\mathrm{K}_{2} \mathrm{O}$

Total

Sample

$\begin{array}{llllll}\text { Core } & 78.05 & 0.16 & 12.57 & 0.79 & 0.03\end{array}$

0.17

0.79

4.08

$3.35 \quad 0.20 \quad 4.35$

At9.15

$\operatorname{Re}$

$\begin{array}{llllll}\text { Core } & 78.10 & 0.14 & 12.65 & 0.78 & 0.03\end{array}$

$0.15 \quad 0.76$

4.03

$\begin{array}{lll}3.37 & 0.19 & 3.93\end{array}$

At9.16

$\operatorname{Re}$

A

$\begin{array}{llllll}\text { Core } & 77.83 & 0.16 & 12.74 & 0.91 & 0.08\end{array}$

$0.14 \quad 0.80$

4.00

$\begin{array}{lll}3.33 & 0.20 & 3.87\end{array}$

At9.18

$\operatorname{Re}$ $\begin{array}{llllll}\text { Core } & 77.88 & 0.16 & 12.65 & 0.97 & 0.09\end{array}$

$0.12 \quad 0.80$

4.12

$\begin{array}{lll}3.20 & 0.19 \quad 4.89\end{array}$

At9.19

$\operatorname{Re}$

A

$\begin{array}{llllll}\text { Core } & 77.63 & 0.15 & 12.71 & 1.08 & 0.08\end{array}$

0.15

0.78

3.90

$\begin{array}{lll}3.52 & 0.21 \quad 3.09\end{array}$

At9.20

$\operatorname{Re}$ $\begin{array}{llllll}\text { Core } & 78.00 & 0.14 & 12.56 & 0.97 & 0.13\end{array}$

0.18

0.80

4.01

$3.22 \quad 0.20 \quad 4.73$

At_10.1

$\operatorname{Re}$

A

$12.72 \quad 0.81 \quad 0.10$

0.19

0.84

3.89

$\begin{array}{lll}3.62 & 0.24 & 5.48\end{array}$

At_10.2

$\operatorname{Re}$

$\operatorname{Re}$ $\begin{array}{llllll}\text { Core } & 78.01 & 0.17 & 12.56 & 0.89 & 0.10\end{array}$

0.16

0.80

4.03

$3.28 \quad 0.20 \quad 3.62$

At_10.4

$\begin{array}{llllll}\text { A } & & & & & \\ \text { Core } & 77.88 & 0.18 & 12.61 & 1.00 & 0.04\end{array}$

0.15

0.81

4.06

$3.27 \quad 0.17 \quad 4.37$

At_10.5

Re

$\operatorname{Re}$ Core $\quad 77.68 \quad 0.15$

A

$12.17 \quad 1.25 \quad 0.05$

0.09

0.68

4.07

$\begin{array}{lll}3.86 & 0.22 \quad 3.19\end{array}$

At_10.6

$\begin{array}{llllll}\text { Core } & 78.04 & 0.16 & 12.56 & 0.95 & 0.10\end{array}$

0.16

$0.78 \quad 3.97$

3.29

$0.21 \quad 5.45$

At_10.7

Re

$\operatorname{Re}$ $\begin{array}{llllll}\text { Core } & 78.18 & 0.17 & 12.65 & 0.84 & 0.11\end{array}$

0.12

A

$\begin{array}{llllll}\text { Core } & 77.87 & 0.14 & 12.62 & 0.90 & 0.09\end{array}$

0.15

$0.80 \quad 3.93$

3.22

At_10.8

Re

A

$\begin{array}{lll}1.62 & 0.90-0.09 \\ 12.52 & 0.89 & 0.05\end{array}$

0.16

0.78

4.02

$3.44 \quad 0.19 \quad 5.23$

At 10.9

$\begin{array}{llllll}\text { Core } & 77.95 & 0.17 & 12.52 & 0.89 & 0.05\end{array}$

A

$\begin{array}{lll}12.47 & 0.99 & 0.12\end{array}$

0.14

0.82

4.1

$3.31 \quad 0.21 \quad 4.25$

At_10.1

Core $\quad 78.12 \quad 0.14$

Re

A

$12.75 \quad 0.79 \quad 0.05$

0.16

0.78

4.05

3.19

At_10.1 Core $\quad 77.82 \quad 0.13$

Re

A

0.19

$\begin{array}{lll}12.56 & 0.88 & 0.10\end{array}$

0.16

$0.75 \quad 4.2$

$\begin{array}{lll}3.19 & 0.19 & 4.73\end{array}$

$2-$

At_10.1

Core $78.06 \quad 0$

Re

A

$12.77 \quad 1.04 \quad 0.13$

$\begin{array}{lll}0.16 & 0.80 \quad 3.86\end{array}$

$3.27 \quad 0.21 \quad 3.85$

At_10.1 Core $\quad 77.79 \quad 0.17$

Re

A

0.17

$\begin{array}{lll}0.16 & 0.82 \quad 3.95\end{array}$

$3.39 \quad 0.20 \quad 5.83$

4

At_10.1

Core $\quad \begin{array}{lllll}78.00 & 0.17 & 12.48 & 0.90 & 0.10\end{array}$

$0.14 \quad 0.82 \quad 4.09$

$\begin{array}{lll}3.18 & 0.18 \quad 3.87\end{array}$

5

At_10.1

Re

A

$12.48 \quad 0.90$

$3.28 \quad 0.18 \quad 4.23$

6

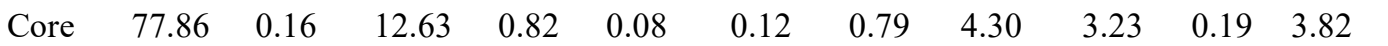

At_10.1

Re

$\begin{array}{ll}\text { A } & \\ \text { Core } & 77.72 \quad 0.16\end{array}$

$\begin{array}{lll}12.67 & 0.83 & 0.14\end{array}$

0.15

0.79

$\begin{array}{llll}4.32 & 3.22 & 0.19 & 4.20\end{array}$

At_10.1

$\mathrm{Re}$

A
Core $\quad 77.76 \quad 0.13$

$\begin{array}{lll}12.76 & 0.91 & 0.10\end{array}$

$0.12 \quad 0.80$

4.1

At 10.1

A

$\begin{array}{llll}0.12 & 12.52 & 0.88 & 0.10\end{array}$

$0.14 \quad 0.80$

4.2

3.23

$\begin{array}{lll}23 & 0.20 & 3.11\end{array}$

9 -

$\mathrm{Re}$

B

78.0

$\begin{array}{llll}0.15 & 12.37 & 0.77 & 0.06\end{array}$

$0.12 \quad 0.78$

3.93

$\begin{array}{lll}3.21 & 0.19 & 3.99\end{array}$

At_10.2

$\operatorname{Re}$

B

$\begin{array}{llll}0.14 & 12.52 & 0.82 & 0.04\end{array}$

$0.15 \quad 0.79$

$\begin{array}{lllll}4.01 & 3.17 & 0.18 & 5.84 & \text { Bt_39.3 }\end{array}$

B

78.36

$\begin{array}{llll}0.14 & 12.56 & 0.93 & 0.08\end{array}$

$0.15 \quad 0.74$

$\begin{array}{lllll}4.10 & 3.36 & 0.18 & 3.49 & \text { Bt_39.4 }\end{array}$

B

$\begin{array}{llll}0.14 & 12.27 & 0.87 & 0.07\end{array}$

$0.14 \quad 0.7$

$\begin{array}{lll}3.95 & 3.15 & 0.19\end{array}$

4.29 Bt_39.5

B

78.70

$\begin{array}{llll}0.18 & 12.68 & 0.94 & 0.04\end{array}$

$0.15 \quad 0.82$

$\begin{array}{lll}3.72 & 3.16 & 0.20\end{array}$

7.53 Bt_39.7

B

78.

$\begin{array}{llll}0.16 & 12.56 & 0.93 & 0.08\end{array}$

$0.15 \quad 0.76$

$\begin{array}{lll}3.79 & 3.12 & 0.1\end{array}$

4.45 Bt_39.8

B

$\begin{array}{llll}0.15 & 12.67 & 0.95 & 0.05\end{array}$

$0.15 \quad 0.80$

$\begin{array}{lll}4.12 & 3.26 & 0.20\end{array}$

3.42 Bt_39.19

B

78.18

$\begin{array}{llll}0.14 & 12.58 & 0.91 & 0.07\end{array}$

$0.14 \quad 0.8$

$\begin{array}{lllll}4.05 & 3.12 & 0.20 & 5.15 & \text { Bt_39.10 }\end{array}$ 
Tephra Bore-

$\mathrm{SiO}_{2}$

Core $\quad 78.05$

$\mathrm{Re}$

Re

$\mathrm{Re}$

Re

Re

$\operatorname{Re}$

Re

Re

Re

Re

Re

Re

Re

Re

Re

Re

Re

Re

Re

Re

Re

Re

Re

Re

Re

Re

Re

Re

$\operatorname{Re}$

Re

$\operatorname{Re}$

\section{B} B

Core $\quad 78.13$ B

Core $\quad 78.06$ B

Core $\quad 77.98$ B

Core $\quad 78.12$

B

Core $\quad 78.31$ B

Core 78.12

B

Core $\quad 78.74$ B

Core $\quad 78.49$

B

Core $\quad 77.92$ B

Core $\quad 78.07$

B

Core $\quad 77.56$ B

Core $\quad 77.80$

B

Core $\quad 78.09$ B Core $\quad 77.84$ B

Core $\quad 77.95$ B Core $\quad 77.86$ B

Core 78.13 B Core $\quad 77.98$ B Core $\quad 77.92$ B Core $\quad 77.99$ B B Core 77.95 B Core $\quad 77.75$ B Core $\quad 77.92$ B B B Core $\quad 78.09$ B B $\begin{array}{ll}\text { Core } & 77.75 \\ \text { B }\end{array}$ Core 78.54 B Core $\quad 77.94$
Core $\quad 78.31$
$0.17 \quad 12.67 \quad 0.86$

$0.13 \quad 12.44 \quad 0.92$

$0.16 \quad 12.58$
0.07

0.05

0.04

$\begin{array}{lll}0.18 & 12.52 & 0.8\end{array}$

$0.18 \quad 12.49$

$0.14 \quad 12.35 \quad 1.01$

$0.18 \quad 12.30$

0.85

$0.14 \quad 12.62 \quad 0.85$

$0.15 \quad 12.37$

0.77

$0.17-12.53$

$0.14 \quad 12.62 \quad 1.00$

$0.16 \quad 12.51$

$0.14 \quad 12.58 \quad 1.00$

$\begin{array}{lll}0.18 & 12.78 & 0.88\end{array}$

$\begin{array}{lll}0.15 & 12.40 \quad 1.05\end{array}$

$\begin{array}{lll}0.17 & 12.68 & 0.90\end{array}$

$0.15 \quad 12.49$

0.88

$0.14 \quad 12.63$

$0.14 \quad 12.51$

0.92

0.93

$0.13 \quad 12.62 \quad 0.82$

$\begin{array}{lll}0.14 & 12.70 \quad 0.86\end{array}$

0.03

0.03

$0.15 \quad 0.78$

$\begin{array}{lll}4.09 & 3.35 & 0.19\end{array}$

$\begin{array}{lll}4.08 & 3.34 & 0.20\end{array}$

$\begin{array}{llll}0.16 & 12.65 & 0.90 & 0.01\end{array}$

$\begin{array}{lll}0.13 & 12.56 \quad 0.93\end{array}$

0.05

0.1

.14

0.77

$\begin{array}{lll}4.09 & 3.29 & 0.18\end{array}$

3.65

Bt_40.1

5

Bt 40.1

0.120 .8

$4.27 \quad 3.36$

$3.36 \quad 0.20$

4.64

Bt 40.1

$\begin{array}{lll}0.14 & 12.60 \quad 0.86\end{array}$

0.05

0.13

0.82

4.07

$\begin{array}{lll}0.19 & 12.76 \quad 0.88\end{array}$

0.06

0.1

0.82

4.20

3.39

0.17

3.13

7

$\begin{array}{lll}0.17 & 12.52 \quad 1.02\end{array}$

0.05

$0.15 \quad 0.80$

$\begin{array}{lll}3.98 & 3.38 & 0.19\end{array}$

0.19

3.85

8

$\begin{array}{lll}0.18 & 12.52 \quad 0.80\end{array}$

0.07

$0.32 \quad 0.73$

3.76

$3.76 \quad 3.33$

$33 \quad 0.55$

.55

4.619

Bt_40.1

$\begin{array}{llll}0.14 & 12.61 & 0.90 & 0.0\end{array}$

$\begin{array}{llll}0.16 & 12.69 & 0.87 & 0.05\end{array}$

$0.12 \quad 0.82$

$\begin{array}{lll}3.95 & 3.33 & 0.20\end{array}$

$\begin{array}{lll}4.25 & 3.28 & 0.19\end{array}$

$19 \quad 4.85 \quad$ Bt 41.3

$\begin{array}{llll}0.16 & 12.56 & 0.80 & 0.03\end{array}$

$0.15 \quad 0.82$

$0.16 \quad 0.7$

$\begin{array}{lll}3.88 & 3.11 & 0.1\end{array}$

5.50 Bt_41.4

$\begin{array}{lll}0.17 & 12.57 \quad 0.91\end{array}$
0.02 $\begin{array}{lllllll}0.13 & 0.84 & 4.15 & 3.27 & 0.18 & 4.92 & \text { Bt } 41.5\end{array}$ 
Tephra

Name

Bore-

hole

$\operatorname{Re}$

Re

Re

Re

$\operatorname{Re}$

Re

$\mathrm{Re}$

$\mathrm{Re}$

$\operatorname{Re}$

$\mathrm{Re}$

$\operatorname{Re}$

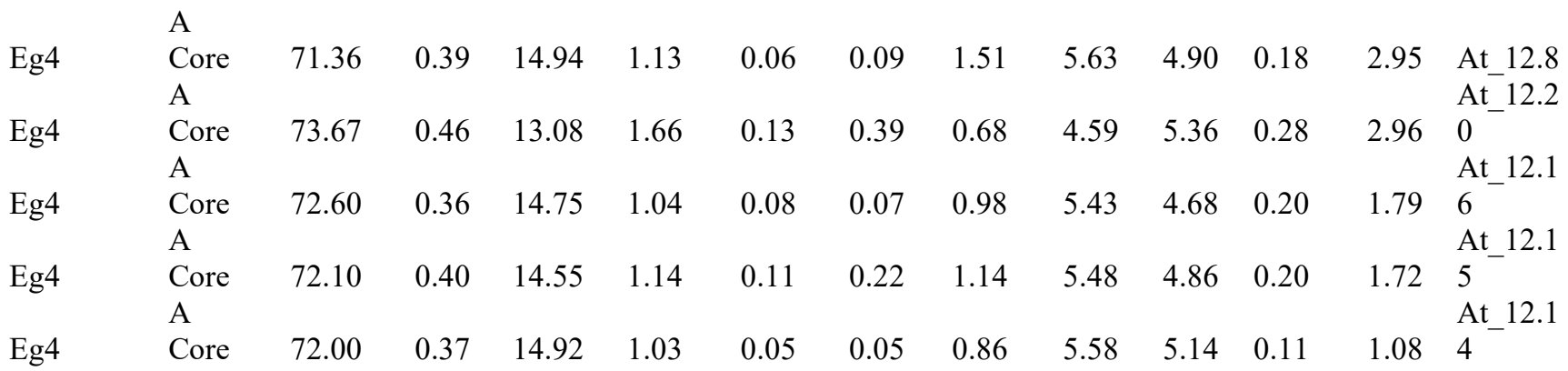

Sample

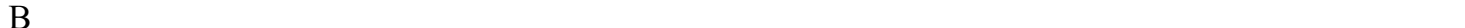

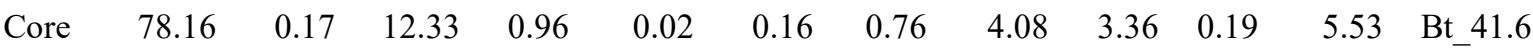
$\begin{array}{lllllllllllll}\text { B } & & & & & & & & & & & & \\ \text { Core } & 77.80 & 0.17 & 12.64 & 0.90 & 0.06 & 0.14 & 0.74 & 4.15 & 3.39 & 0.19 & 1.84 & \text { Bt_41.7 }\end{array}$ B $\begin{array}{lllllllllllll}\text { Core } & 78.00 & 0.14 & 12.60 & 0.90 & 0.05 & 0.16 & 0.79 & 4.09 & 3.28 & 0.18 & 4.28 & \text { Bt_41.8 } \\ \text { B } & & & & & & & & & & & & \text { Bt_41.1 }\end{array}$ $\begin{array}{lllllllllllll}\text { Core } & 77.85 & 0.16 & 12.54 & 0.92 & 0.05 & 0.15 & 0.81 & 4.35 & 3.17 & 0.19 & 4.28 & 0\end{array}$

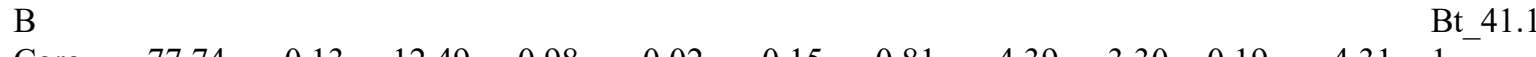
$\begin{array}{lllllllllllll}\text { Core } & 77.74 & 0.13 & 12.49 & 0.98 & 0.02 & 0.15 & 0.81 & 4.39 & 3.30 & 0.19 & 4.31 & 1\end{array}$ $\begin{array}{lllllllllllll}\text { B } & & & & & & & & & & & & \\ \text { Core } & 78.01 & 0.15 & 12.64 & 0.88 & 0.05 & 0.16 & 0.88 & 4.05 & 3.17 & 0.18 & 3.30 & 3\end{array}$ B

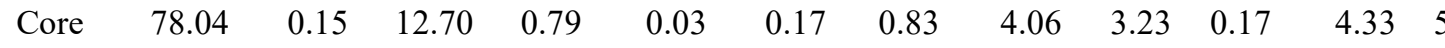
$\begin{array}{lllllllllllll}\text { B } & & & & & & & & & & & \\ \text { Core } & 77.85 & 0.18 & 12.76 & 0.80 & 0.04 & 0.16 & 0.78 & 4.22 & 3.21 & 0.17 & 1.08 & 6\end{array}$

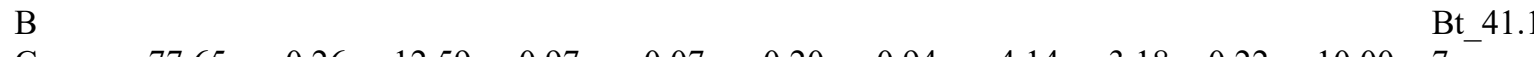

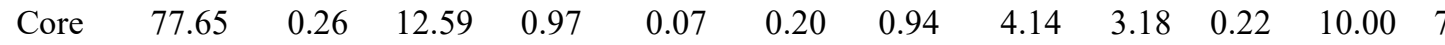
$\begin{array}{llllll}\text { B } & \text { Bt_41.1 }\end{array}$ $\begin{array}{lllllllllllll}\text { Core } & 78.57 & 0.16 & 12.51 & 0.88 & 0.04 & 0.14 & 0.77 & 4.03 & 2.92 & 0.18 & 4.47 & 8\end{array}$ $\begin{array}{lllllllllllll}\text { B } & & & & & & & & & & & & \\ \text { Bt_41.1 }\end{array}$

Bt_41.1 Eg4 